\title{
Red de sensores inteligentes para adquisición de datos de una planta de desalinización de agua
}

\section{Smart sensor network for data acquisition of a water desalination plant}

\author{
Jorge Valdez, jcvaldez@uaco.unpa.edu.ar \\ Daniel Pandolfi, dpandolfi@uaco.unpa.edu.ar \\ Andrea Villagra, avillagra@uaco.unpa.edu.ar \\ LabTEm (Laboratorio de Tecnologías Emergentes) \\ Universidad Nacional de la Patagonia Austral. Unidad Académica Caleta Olivia \\ Ruta N ${ }^{\circ} 3$ Acceso Norte, Caleta Olivia, Santa Cruz, Argentina
}

Recibido: 22/05/2018. Aceptado: 01/08/2018

\begin{abstract}
RESUMEN
Una red de sensores inalámbricos (en inglés Wireless Sensor Network, WSN) es un conjunto de nodos sensores y actuadores desplegados sobre la región de actuación. Los sistemas SCADA (Supervisión, Control y Adquisición de Datos) se aplican a la administración de la recolección de información de los procesos de controles industriales, tales como las redes eléctricas, redes de distribución de agua, transporte, etc. Los sistemas Multi-Agente (en inglés Multi-Agent System, MAS) aparecieron como un nuevo enfoque arquitectónico en aplicaciones de ingeniería complejas y altamente dinámicas. Además, los MAS pueden incluir agentes reactivos como Arduinos y agentes inteligentes tales como Redes Neuronales Artificiales, o Lógica Difusa. En este trabajo, se propone construir un sistema tipo SCADA flexible e interoperable, basado en la integración de protocolos de arquitecturas MAS. El sistema propuesto será implementado para administrar un sistema de adquisición de datos y control de operación de tipo SCADA, flexible e interoperable, basado en la integración de los protocolos de procesos MAS y Arduino para la adquisición de datos de una planta de desalinización de agua de mar, de tipo humidificación/des-humidificación, con capacidad de producir $0,1-0,5 \mathrm{~m}^{3} / \mathrm{h}$ de agua dulce. Particularmente en este trabajo se discute la red de sensores para adquisición de datos y detalles de implementación del mismo.
\end{abstract}

Palabras clave: Red de sensores inalámbricos, agentes inteligentes, sistemas multi-agentes, Arduino.

\begin{abstract}
A Wireless Sensor Network (WSN) is a set of sensor and actuator nodes deployed on the region of action. The SCADA systems (Supervision, Control and Data Acquisition) are applied to the administration of information collection of industrial control processes, such as electricity grids, water distribution networks, transportation, etc. The Multi-Agent System (MAS) appeared as a new architectural approach in complex and highly dynamic engineering applications. In addition, MAS can include reactive agents such as Arduinos and intelligent agents such as Artificial Neural Networks or Fuzzy Logic. In this work, we make the propose to build a flexible and interoperable SCADA type system, based on the integration of MAS architecture protocols. The proposed system will be implemented to
\end{abstract}


administer a flexible, interoperable SCADA-type data acquisition and operation control system, based on the integration of the MAS and Arduino process protocols for the data acquisition of a desalination plant of water sea, by humidification / de-humidification process, with the capacity to produce $0.1-0.5 \mathrm{~m}^{3} / \mathrm{h}$ of fresh water. Particularly, in this work the sensor network for data acquisition and implementation details thereof is discussed.

Keywords: Network of Wireless Sensors, intelligent agents, multi-agent systems, Arduino.

\section{INTRODUCCIÓN}

Los sensores son dispositivos económicos, de bajo consumo de energía con recursos limitados Ahsan et al. (2010). Una WSN se compone de gran número de nodos de sensores con capacidad limitada de computación, almacenamiento y comunicación. Los ambientes, donde se despliegan los nodos sensores, pueden ser aplicados en ambientes controlados (tales como el hogar, oficina, almacén, bosque, etc.) o no controlados (tales como áreas hostiles o de desastre, regiones tóxicas, etc.). Las WSN, representan una de las áreas de investigación más promisorias debido al completísimo campo de aplicación y el desarrollado de nuevas tecnologías de controladores electrónicos o electromecánicos, y los avances en la tecnología de comunicación inalámbrica Charlebois (2004). El diseño de una WSN debe hacer frente a la ocurrencia de defectos de alguna o múltiples componentes en la red y por lo tanto es necesaria la aplicación de técnicas robustas que puedan tolerar los fallos, sin resentir el buen funcionamiento de la red. Los problemas y las necesidades de mecanismos de mejora de la fiabilidad dependen de los recursos y aplicaciones disponibles para el que se aplica la WSN.

Las tecnologías de medición inteligente (SM, en inglés Smart Metering) se han convertido en un tema de importancia creciente no solo en el ámbito científico sino en la industria; se implementan nuevas normativas para su aplicación, se desarrollan proyectos pilotos y han llegado al mercado nuevos dispositivos. Los medidores inteligentes y el análisis avanzado de datos permiten una nueva era de uso de los big data continuos generados por un conjunto muy grande de medidores para crear un sistema inteligente de gestión de aguas Nguyen et al. (2018). La medición inteligente no debe verse como un fin en sí misma, sino que tiene el potencial de satisfacer las necesidades de información sobre el suministro y el uso final, que a su vez puede satisfacer objetivos sostenibles de gestión del agua Boyle et al. (2013). Las empresas de distribución de agua están aprovechando el potencial de los medidores inteligentes para crear patrones diurnos de demanda diaria que reflejen las características de consumo de desarrollos individuales que cada vez aplican más fuentes de agua alternativas y dispositivos ultra-eficientes Gurung et al. (2015).

Los rápidos avances en las tecnologías electrónicas han dado lugar al desarrollo de una variedad de nuevas y baratas placas electrónicas con capacidades de detección, monitoreo y control que ofrecen oportunidades para su implementación en múltiples áreas de aplicación. La placa Arduino se desarrolló originalmente en el año 2005 en el Interaction Design Institute, de Ivrea Italia, como una plataforma de hardware de código abierto. Estudios sobre Arduino, muestran su utilidad en las mediciones automatizadas, y ofrecen una guía para otros investigadores en el desarrollo de sensores de bajo costo y sistemas de monitoreo y automatización en diversos campos de aplicación Zhang (2009).

Según Abbas et al. (2015), un sistema SCADA es responsable de recopilar información y datos en tiempo real de la variedad de procesos o ambiente y proporcionar estos datos a los operadores situados en cualquier lugar y en cualquier momento. Desafortunadamente, muchos de los sistemas SCADA convencionales no son capaces de proporcionar la gestión 
de la información y enfoques inteligentes de alto nivel. Los MAS proponen soluciones a problemas distribuidos en los dominios computacionales dinámicos y abiertos. Para Melgar y Diez (2012), los sistemas informáticos, especialmente los relacionados con las aplicaciones industriales modernas, tales como los sistemas SCADA, se están volviendo cada vez más interconectados y más difícil en mantener. Los enfoques basados en agentes (MAS) parecen ser una solución prometedora y el rápido desarrollo del campo de los sistemas basados en agentes ofrece un nuevo paradigma interesante para el desarrollo de programas sofisticados en entornos dinámicos y abiertos.

Debido a los grandes avances realizados en las tecnologías RFID (Identificación por Radio Frecuencia) y WSN, se ha estudiado en varios tipos de aplicaciones que involucran vehículos, tales como el pago automático de peaje, gestión de flotas, seguridad de la navegación y sistemas de transporte inteligentes. Además, para mejorar la eficiencia y la seguridad en los sistemas de transporte, se está utilizando tecnología RFID en diferentes tipos de aplicaciones tales como estacionamiento inteligente, los billetes electrónicos, el cobro de peajes, seguimiento de la ruta virtual y control de semáforos digital. La tecnología RFID se puede utilizar en la identificación automática de vehículos para obtener información en tiempo real de las rutas, y simplemente consiste en colocar etiquetas $R F I D$ pasivas en varios lugares de la ruta elegida, y lectores RFID en el autobús. Araar y Khali (2012), propusieron la incorporación de las tecnologías RFID y WSN con el fin de identificar y rastrear vehículos sustituyendo sistemas basados en GPS (Sistema de Posicionamiento Global).

El uso de WSNs para la auditoría y la gestión del consumo de energía en un edificio es un área de investigación emergente Guerrieri et al. (2011). En Narayan et al. (2015), y Hani y Koiv (2012) se presentan distintos sistemas de control y gestión para la conservación de la energía en grandes edificios públicos diseñado para conectar el sistema de medición de distribución de energía, una red de sensores inalámbricas, y la automatización de edificios. Corucci et al. (2011), proponen GreenBuilding, un sistema basado en sensores para la administración de energía automatizada de aparatos eléctricos en un edificio capaz de proporcionar un importante ahorro energético mediante el uso de estrategias de conservación de la energía apropiados adaptados a aparatos específicos.

Las WSNs han emergido como una tecnología eficaz para el control de la infraestructura crítica, tales como tuberías de agua, petróleo y gas. En Almazyad et al. (2014), se muestra un diseño escalable y la simulación de un sistema de monitoreo de fugas de tuberías de agua utilizando la RFID y tecnología WSN. Huang et. al (2015), proponen un enfoque viable y eficaz en el uso de una WSN para monitorear la calidad del agua de un lago y además se propone un motor de razonamiento basado en reglas que se utiliza para llevar acabo un sistema de soporte de decisiones a través de técnicas de razonamiento y sensibilidad al contexto. Luck (1999) propone uno de los primeros WSN, un sistema de monitoreo ambiental del agua basado en sensores inalámbricos. Esta WSN se compone de tres partes: un nodo de control de datos, base de datos y un centro de monitorización remota.

El sistema de adquisición de datos propuesto para la gestión de una WSN está basado en una arquitectura MAS y permite el monitoreo y control de una planta de desalación. Tanto el hardware como el software son propuestos están basados en tecnologías Open Source y son descriptos a continuación. El artículo está organizado de la siguiente manera: la Sección 2 presenta información sobre el sistema basado en la humidificación y des-humidificación del aire, la Sección 3 se presenta el sistema propuesto para la adquisición de datos. Finalmente, la Sección 4 presenta una discusión acerca del trabajo y posibles trabajos futuros y finalmente se presentan las conclusiones. 


\section{PLANTA DE DESALACIÓN DE AGUA}

Los sistemas desarrollados hasta la actualidad para la desalinización de agua salobre o de mar se pueden clasificar en dos tipos básicos: los que se basan en un proceso térmico, ya sea utilizando combustibles fósiles o energía solar para calentar el agua salada, y aquellos que utilizan membranas y alta presión para realizar un proceso de "filtrado" de las sales disueltas. A continuación, realizaremos una breve descripción de los procesos utilizados en desalinización, basados en la tecnología de Humidificación / Des-humidificación.

El equipo que se está construyendo se basa en el ciclo natural del agua, Ma et al. (2010) y consta, básicamente, de tres secciones:

- Un humidificador de aire.

- Un condensador de la humedad que este aire húmedo acarrea.

- Un equipo de calentamiento del agua de mar.

Esquemáticamente se puede observar en la figura 1. Sobre una torre de humidificación se distribuye desde la parte superior agua salada a una temperatura de $85{ }^{\circ} \mathrm{C}-95^{\circ} \mathrm{C}$. Parte del agua se evapora a medida que fluye hacia abajo y se va enfriando. Al mismo tiempo, cierta cantidad de aire seco fluye en contra corriente, ya sea por convección natural o forzado, ganando humedad relativa a medida que se pone en contacto con el agua. El aire se humedece a medida que se calienta en su curso ascendente. La cantidad de humedad que es capaz de absorber aumenta notablemente con la temperatura. En el condensador fluye agua de mar fría, intercambiando calor con el aire húmedo y caliente que sale del humidificador. La humedad contenida en el aire se condensa a medida que este se enfría en el condensador y se escurre hacia el fondo, donde es recolectada como agua dulce.

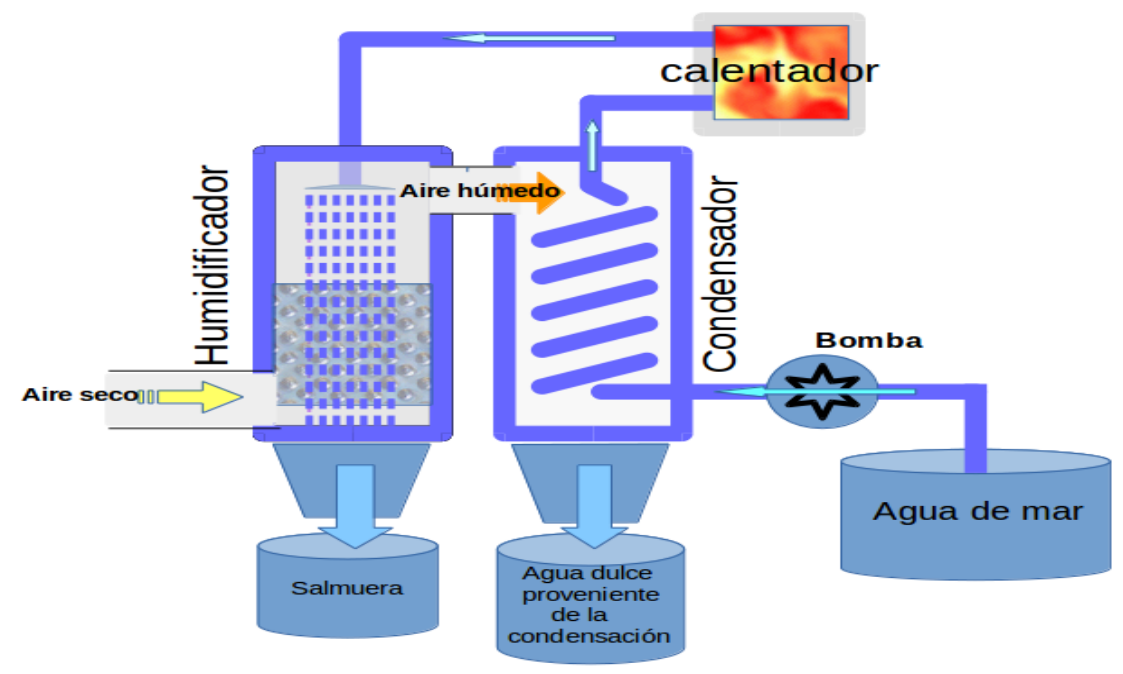

Figura 1: Modelo esquemático de una planta de desalinización por humidificación/des-humidificación con sus diversas componentes.

El agua de mar que se utiliza para enfriar el aire sale del condensador a una cierta temperatura, que es superior a la de entrada, pues intercambió calor con el aire húmedo. Su temperatura no llega a los $85-95^{\circ} \mathrm{C}$ que necesita para ingresar al humidificador y por este motivo se debe recalentar con una fuente de calor externa, ya sea una caldera a base de combustible fósil, con energía solar en zonas tropicales, etc. El agua que sale por debajo del humidificador mantiene la misma cantidad absoluta de sales, pero perdió agua pura, por lo cual su salinidad ha aumentado. Esta salmuera es colectada en el fondo de la sección del humidificador y se vierte nuevamente al mar. 
El equipo se está construyendo en la Unidad Académica Caleta Olivia de la Universidad Nacional de la Patagonia Austral en el marco de un Proyecto de Desarrollo Tecnológico y Social (PDTS). Hasta el presente, se ha construido la sección de humidificación del aire y se está testeando en condiciones de laboratorio. Esta consta de una torre de humidificación de $40 \mathrm{~cm}$ de diámetro, construida en acero galvanizado, con empaque random conformado por anillos tipo pall de acero inoxidable de 3/4". La torre fue diseñada para que funcione por convección natural del aire. El calentador que se está empleando es una pequeña caldera industrial del tipo utilizado para calefacción domiciliaria con radiadores. Las pruebas se están llevando a cabo con agua de red, ya que sus propiedades termodinámicas no difieren sustancialmente de las del agua de mar. Posteriormente, una vez concluidas las pruebas y completada la construcción de la planta piloto, se emplazará en la planta de desalinización por ósmosis inversa, donde se la hará funcionar con agua de mar en las condiciones locales. Se ha diseñado un condensador de vapor de tipo coraza y tubos el cual se está construyendo. Los cálculos preliminares muestran que este es un dispositivo crítico para el buen funcionamiento de la planta.

\section{SISTEMA DE AQUISICIÓN DE DATOS PROPUESTO}

En cuanto a los dispositivos de adquisición de datos y control, estos estarán operados por un sistema SCADA, Venkatesan et al. (2013), (Supervisión, Control y Adquisición de Datos) con el objeto facilitar la retro-alimentación en tiempo real del equipo a través de actuadores. El sistema propuesto propone las siguientes ventajas: 1) es simple de operar; 2) es flexible; 3 ) es interoperable; 4) es de bajo costo y fuente abierta. Todos los dispositivos de adquisición de datos (anemómetros, caudalímetros, termómetros y sensores de consumo de gas y de energía eléctrica) serán operados con estos protocolos. Para ello se han desarrollado softwares específicos y se están montando las consolas apropiadas para acoplarlas a la planta. Ya se han realizado las primeras mediciones que permitieron obtener los parámetros de la torre de humidificación e información importante para el diseño del intercambiador de calor, que provea la mayor recuperación posible de energía junto con la condensación de la mayor cantidad de agua posible.

El Sistema de adquisición de datos estará conformado por diversas tecnologías de hardware y software. A continuación, se describirán cada una de las arquitecturas involucradas:

\subsection{Arquitectura de hardware}

El hardware empleado constará de los siguientes dispositivos:

Unidad de procesamiento: Una computadora Raspberry Pi 3 [28]. La Raspberry Pi 3 es la tercera generación, este remplazó a la Raspberry Pi Modelo B en febrero de 2016. En la Tabla 1 se puede observar las especificaciones técnicas.

Los dispositivos de hardware Arduino y los sensores permitirán el sensado de cada operación de la planta de desalinización. Se definieron tres unidades de sensado, cada uno de los cuales se detallan a continuación:

Unidad de sensado para la medición de temperatura, humedad y caudal de aire húmedo, Callaway (2004). (En la figura 2 puede verse el diagrama de conexión):

- Placa Arduino Mega (las especificaciones pueden observarse en la Tabla 2) Caffery y Gordon (2010),

- Sensor de Temperatura y Humedad (con protección) SHT20 de la empresa Sensirion (las especificaciones pueden observarse en las Tablas 3 y 4 ),

- Sensor de Presión Diferencial MPX5500DP de NXP (las especificaciones pueden observarse en la Tabla 5) 
Unidad de sensado para la medición de temperatura, humedad y caudal de aire seco:

- Placa Arduino Mega,

- Sensor de Temperatura y Humedad (con protección) SHT20 de la empresa Sensirion,

- Sensor de Presión Diferencial MPX5500DP de NXP.

Unidad de sensado para la medición de caudal de agua caliente (en la Figura 3 puede observarse el diagrama de conexión):

- Placa Arduino Mega

- Sensor de caudal YF-S201

Todas las unidades de sensado se conectaron a la computadora Raspberry Pi 3 mediante los siguientes elementos:

- HUB USB de 10 puertos 2.0 con fuente externa.

- $\quad$ Cables USB 2.0

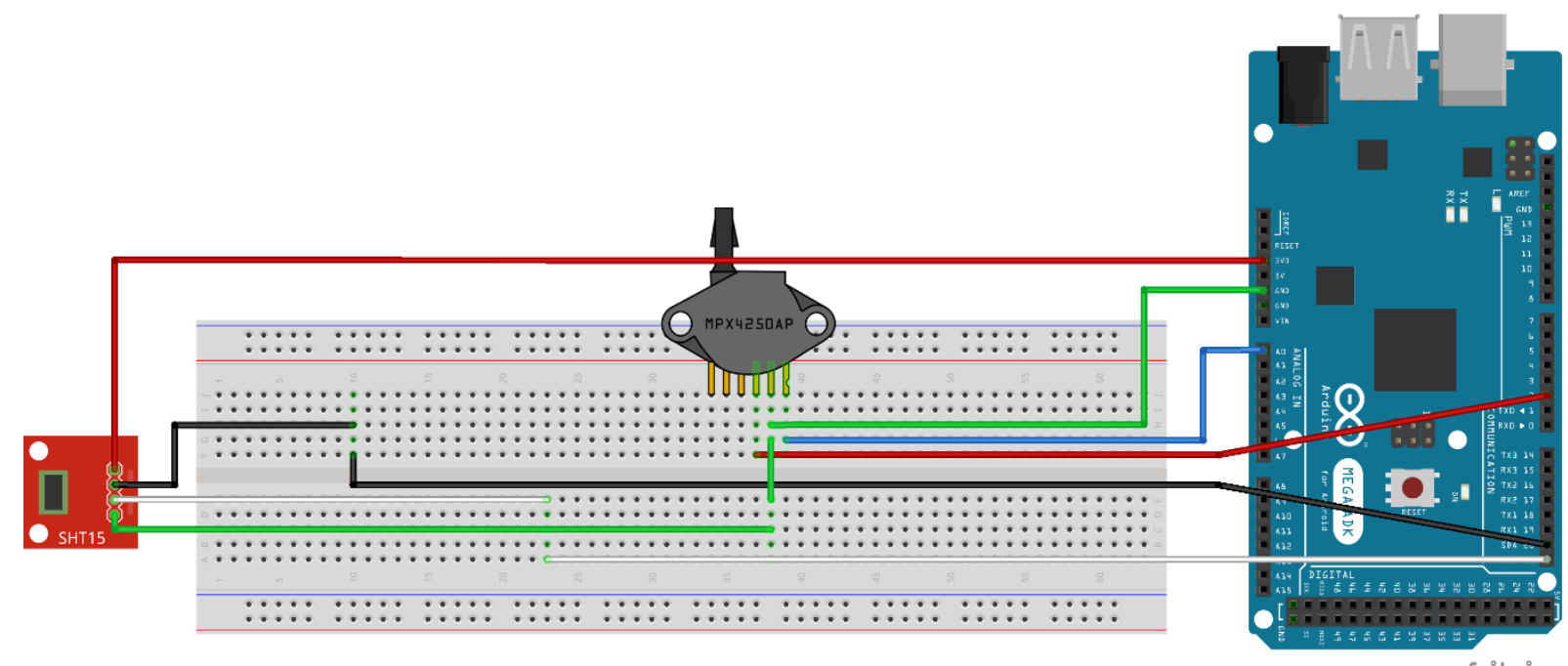

Figura 2: Diagrama de conexión de la Unidad de sensado de caudal de aire (húmedo y seco) y temperatura.

- CPU con 4 núcleos de 64 Bits ejecutándose a 1.2GHz Broadcom BCM2837 64bit

- Memoria DDR3 de 1GB

- Tarjeta Inalámbrica (Wireless y Bluetooth) BCM43438 de Bajo Consumo integrada en

la placa

- Puerto de expansión de 40 pines GPIO

- 4 Puertos USB

- Salida estéreo de 4 polos y puerto de vídeo compuesto

- Conector HDMI

- Puerto de cámara CSI para conectar una cámara Raspberry Pi

- DSI Display Port para conectar una pantalla táctil Raspberry Pi

- Puerto para tarjeta Micro SD para cargar el sistema operativo y almacenar datos

- Fuente de alimentación conmutada Micro USB mejorada hasta 2.5A

Tabla 1: Especificaciones técnicas Raspberry Pi 3 Modelo B. 


\begin{tabular}{|l|l|}
\hline Microcontrolador & ATMega2560 \\
\hline Voltaje de Operación & $5 \mathrm{~V}$ \\
\hline Voltaje de Entrada (Recomendado) & $7-12 \mathrm{~V}$ \\
\hline Voltaje de Entrada (Limite) & $6-20 \mathrm{~V}$ \\
\hline Pines Digitales E/S & $\begin{array}{l}54(\mathrm{de} \text { los cuales 15 proveen salida } \\
\text { PWM) }\end{array}$ \\
\hline Pines Análogos de Entrada & 16 \\
\hline Corriente por Pin E/S & $20 \mathrm{~mA}$ \\
\hline Corriente para Pin de 3.3V & $50 \mathrm{~mA}$ \\
\hline Memoria Flash & $256 \mathrm{~KB}$ de los cuales 8 KB son usados \\
& por el Bootloader \\
\hline SRAM & $8 \mathrm{~KB}$ \\
\hline EEPROM & $4 \mathrm{~KB}$ \\
\hline Velocidad del Reloj & $16 \mathrm{MHZ}$ \\
\hline LED onboard & 13 \\
\hline Largo & $101.52 \mathrm{~mm}$ \\
\hline Ancho & $53.3 \mathrm{~mm}$ \\
\hline Peso & $37 \mathrm{~g}$ \\
\hline
\end{tabular}

Tabla 2: Especificaciones técnicas Placa Arduino Mega 2560.

\begin{tabular}{|l|c|c|c|}
\hline \multicolumn{1}{|c|}{ Parametros } & Condición & Valor & Unidades \\
\hline \multirow{2}{*}{ Resolución } & 12 bit & 0.04 & $\% \mathrm{RH}$ \\
\cline { 2 - 4 } & $8 \mathrm{bit}$ & 0.7 & $\% \mathrm{RH}$ \\
\hline \multirow{2}{*}{ Tolerancia de Precisión } & Tip & +-3.0 & $\% \mathrm{RH}$ \\
\cline { 2 - 4 } & Max & ver datasheet & $\% \mathrm{RH}$ \\
\hline Repetibilidad & & +-0.1 & $\% \mathrm{RH}$ \\
\hline Histéresis & & +-1 & $\% \mathrm{RH}$ \\
\hline No linealidad & & $<0.1$ & $\% \mathrm{RH}$ \\
\hline Tiempo de respuesta & t $63 \%$ & 8 & $\mathrm{~s}$ \\
\hline Rango de operación & extendido & $0 \mathrm{a} 100$ & $\% \mathrm{RH}$ \\
\hline Deriva a largo plazo & Tip. & $<0.25$ & $\% \mathrm{RH}$ \\
\hline
\end{tabular}

Tabla 3: Especificaciones técnicas sensor Sensirion SHT20, medición de humedad.

\begin{tabular}{|l|c|c|c|}
\hline \multicolumn{1}{|c|}{ Parametros } & Condición & Valor & Unidades \\
\hline \multirow{2}{*}{ Resolución } & 14 bit & 0.01 & ${ }^{\circ} \mathrm{C}$ \\
\cline { 2 - 4 } & 12 bit & 0.04 & ${ }^{\circ} \mathrm{C}$ \\
\hline \multirow{2}{*}{ Tolerancia de Precisión } & Tip & +-0.3 & ${ }^{\circ} \mathrm{C}$ \\
\cline { 2 - 4 } & $\mathrm{Max}$ & ver datasheet & ${ }^{\circ} \mathrm{C}$ \\
\hline Repetibilidad & & +-0.1 & ${ }^{\circ} \mathrm{C}$ \\
\hline Tiempo de respuesta & $\mathrm{t} 63 \%$ & 5 a 30 & ${ }^{\circ} \mathrm{S}$ \\
\hline Rango de operación & extendido & -40 a 125 & ${ }^{\circ} \mathrm{C}$ \\
\hline Deriva a largo plazo & Tip. & $<0.02$ & ${ }^{\circ} \mathrm{C} / \mathrm{yr}$ \\
\hline
\end{tabular}

Tabla 4: Especificaciones técnicas sensor Sensirion SHT20, medición de temperatura. 


\begin{tabular}{|c|c|c|c|c|c|}
\hline Caracteristicas & Simbolo & Min & Tipica & Max & Unidades \\
\hline RepeRango de presión & $\mathrm{P}_{\mathrm{OP}}$ & 0 & - & 500 & $\mathrm{kPa}$ \\
\hline Tensión de alimentación & $\mathrm{V}_{\mathrm{S}}$ & 4.75 & 5.0 & 5.25 & $\mathrm{Vdc}$ \\
\hline Corriente de suministro & $\mathrm{I}_{\mathrm{O}}$ & - & 7.0 & 10 & mAdc \\
\hline Desplazamiento de presión cero $\left(0\right.$ to $\left.85^{\circ} \mathrm{C}\right)$ & $\mathrm{V}_{\text {off }}$ & 0.088 & 0.20 & 0.313 & $\mathrm{Vdc}$ \\
\hline Salida a escala completa & $\mathrm{V}_{\mathrm{FSO}}$ & 4.587 & 4.70 & 4.813 & $\mathrm{Vdc}$ \\
\hline Escala completa & $\mathrm{V}_{\mathrm{FSS}}$ & - & 4.50 & - & $\mathrm{Vdc}$ \\
\hline$\left(0\right.$ to $\left.85^{\circ} \mathrm{C}\right)$ & - & - & - & +-2.5 & $\% \mathrm{~V}_{\mathrm{FSS}}$ \\
\hline Sensibilidad & $\mathrm{V} / \mathrm{P}$ & - & 9.0 & - & $\mathrm{mV} / \mathrm{kPa}$ \\
\hline Tiempo de respuesta & $t_{R}$ & - & 1.0 & - & $\mathrm{ms}$ \\
\hline $\begin{array}{l}\text { Corriente de la fuente de salida a la salida de } \\
\text { escala completa }\end{array}$ & $\mathrm{I}_{\mathrm{O}+}$ & - & 0.1 & - & mAdc \\
\hline Tiempo de calentamiento & - & - & 20 & - & $\mathrm{ms}$ \\
\hline
\end{tabular}

Tabla 5: Especificaciones técnicas sensor de presión diferencial NXP Freescale MPX5500DP.

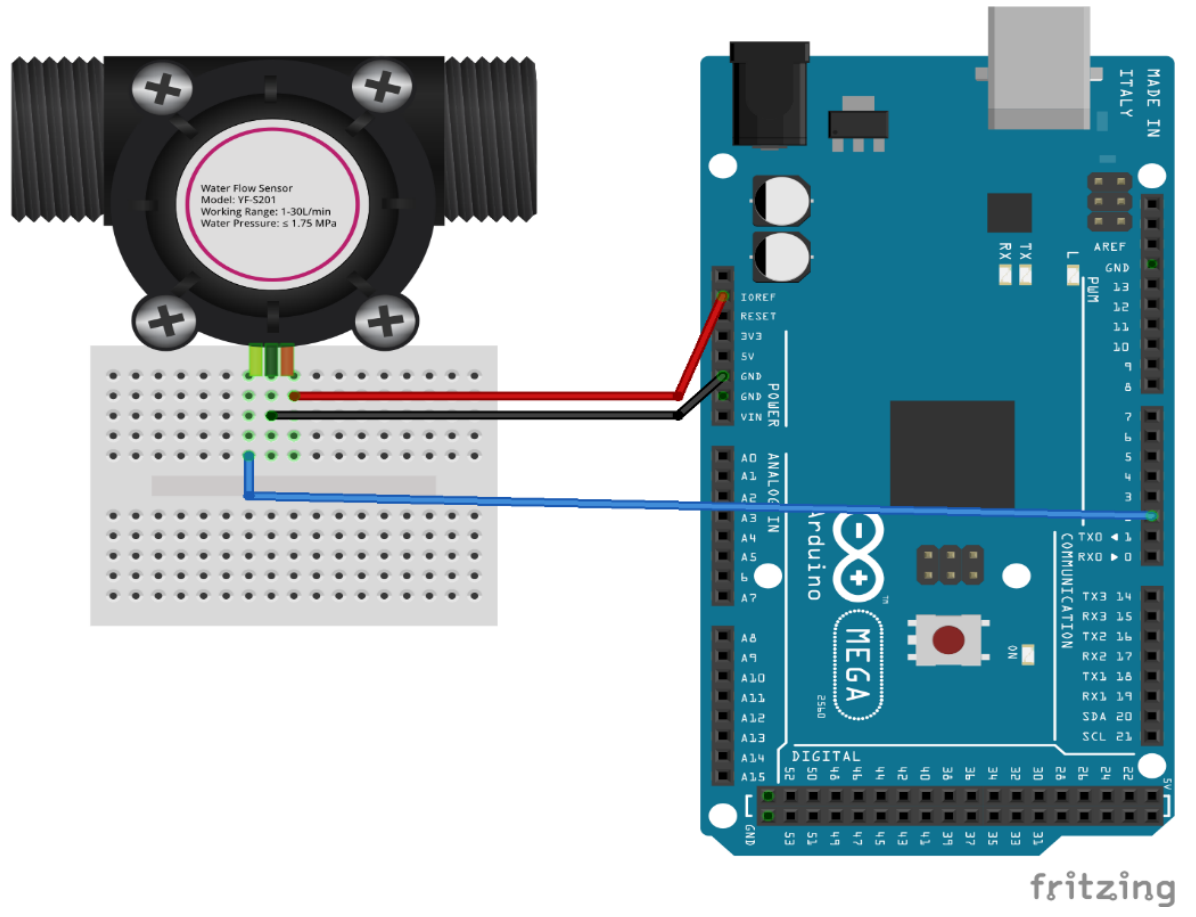

Figura 3: Diagrama de conexión de la Unidad de sensado de caudal de agua.

\subsection{Software base}

El software que se emplea en la computadora Raspberry Pi 3 es la distribución del sistema operativo Linux denominada Raspbian. A continuación, se detallan sus características:

- Nombre de versión: "Stretch",

- Fecha de liberación: 16/08/2017

- Versión del Kernel: 4.9

El lenguaje de programación empleado para manejar los sensores es el empleado por los dispositivos Arduino, el cual es un lenguaje de programación muy similar a Processing Barret (2013). Los scripts Arduino fueron desarrollados empleando el Arduino IDE versión 1.8.2. 


\subsection{Arquitectura de software}

Para el sistema de adquisición de datos, se emplea una plataforma MAS desarrollada empleando un framework de desarrollo denominado SPADE (Smart Python multi-Agent Development Environment), Englund y Wallin (2004). SPADE, es una plataforma destinada a resolver los inconvenientes de los modelos de comunicación de otras plataformas. La plataforma MAS SPADE está construida en torno de un nuevo framework de comunicación, Jiang et al. (2009) que proporciona nuevas poderosas capacidades a la capa de comunicación. Actualmente el protocolo de comunicación que emplea SPADE se denomina XMPP (anteriormente Jabber), Extensible Messaging and Presence Protocol. Es un protocolo abierto y extensible basado en XML, empleado originalmente para mensajería instantánea. En resumidas cuentas, permite el intercambio de mensajes empleando XML. Es ampliamente usado en desarrollos de aplicaciones de mensajería de código abierto. En los últimos años diversas empresas los han adoptado: Facebook, Tuenti, Whatsapp, entre otras.

Adicionalmente esto y para afianzar la garantía de comunicación entre los sensores se empleará el framework de desarrollo denominado ROS, Quigley et al. (2009), el cual significa en sus siglas en ingles Robot Operating System, Schwieren y Vossen (2009), o sistema operativo de robot, empleado hoy en día en diversos grupos de investigación y empresas de la industria robótica. ROS es una plataforma de desarrollo open source para sistemas robóticos. Proporciona toda una serie de servicios y librerías que simplifican considerablemente la creación de aplicaciones complejas para robots. Posee protocolos de comunicación con los principales ambientes de hardware como por ejemplo Arduino. De esta forma se evitó generar protocolos de comunicación necesarios para el intercambio de información entre las unidades de sensado y la computadora Raspberry Pi. ROS permite el uso de distintos lenguajes de programación. De forma oficial soportan Python, C++, Lisp y Java. ROS puede ser ejecutado sobre maquinas tipo Unix, principalmente Ubuntu y Mac OS X. En Microsoft Windows se encuentra en fase experimental.

ROS consta de tres niveles de conceptos:

El nivel de sistema de archivos (Recurso encontrados en el mismo programa):

- Paquetes: Unidad principal para organizar software en ROS.

- Un paquete puede contener procesos ejecutables (nodos), una biblioteca dependiente, conjuntos de datos, archivos de configuración.

- Manifiestos: proporcionan metadatos sobre un paquete, incluyendo su información de licencia y dependencias, así como información específica del compilador.

- Pilas: Es una colección de paquetes que tienen una misma función.

- Manifiestos de pilas: proporcionan datos sobre una pila, incluyendo su información de licencia y sus dependencias en otras pilas.

- Mensajes: definen las estructuras de datos para los mensajes enviados en ROS.

- Servicios: definen la solicitud y estructuras de datos de respuesta de los servicios requeridos por ROS.

El nivel de computación gráfica (conceptos básicos son nodos, maestro, mensajes y temas):

- Nodos: Los nodos son procesos que llevan a cabo cálculos.

- Maestro: El Maestro proporciona registro de nombres y la búsqueda para el resto de la Computación Gráfica. Sin el Maestro, los nodos no serían capaces de encontrar mensajes entre sí, intercambiar, o invocar los servicios.

- Mensajes: Los nodos se comunican entre sí pasando mensajes. Un mensaje es simplemente una estructura de datos que comprende los tipos de campos.

- Temas: Los mensajes se en-rutan a través de un sistema de transporte de publicación/suscripción semántica. Un nodo envía un mensaje por publicar a un determinado tema. El tema es un nombre que se utiliza para identificar el contenido 
del mensaje. Un nodo que está interesado en un determinado tipo de datos se suscribe al tema correspondiente. Puede haber varios editores y suscriptores concurrentes a un mismo tema, y un único nodo puede publicar y/o suscribirse a múltiples temas.

El nivel comunitario: Los conceptos comunitarios ROS Nivel ROS son recursos que permiten a las comunidades el intercambio del software y del conocimiento (distribución, repositorio, wikis, foros).

En la Figura 4 puede observarse la arquitectura de software que tendrá el proyecto. Cada proceso tendrá asociado una unidad de sensado. El núcleo de la plataforma está diseñado sobre esta capa, que comunica los elementos de la plataforma entre sí.

Para el presente trabajo se definieron los siguientes procesos que tendrá asociado el SCADA:

- Proceso de configuración de los datos experimentales, los cuales se denominan Parámetros ROS.

- Proceso de medición de caudal, datos de temperatura, humedad del aire en la entrada de aire seco del humidificador.

- Proceso de medición de caudal, datos de temperatura, humedad del aire en la salida de aire húmedo del humidificador.

- Proceso de medición de temperatura del agua en la entrada de agua caliente del humidificador.

- Procesos de almacenamiento de los datos de las mediciones, se empleará el motor de base de datos de código abierto como lo es MariaDB.

- Proceso de administración de la interfaz de usuario, la misma se implementará mediante un servidor Web.

Los procesos descriptos anteriormente podrían llegar a tener varias instancias, puede requerirse un número mayor de unidades de sensado.

Cada proceso será administrado por un agente, en algunos casos, como por ejemplo la configuración de los datos experimentales no se necesitará tener asociado alguno.

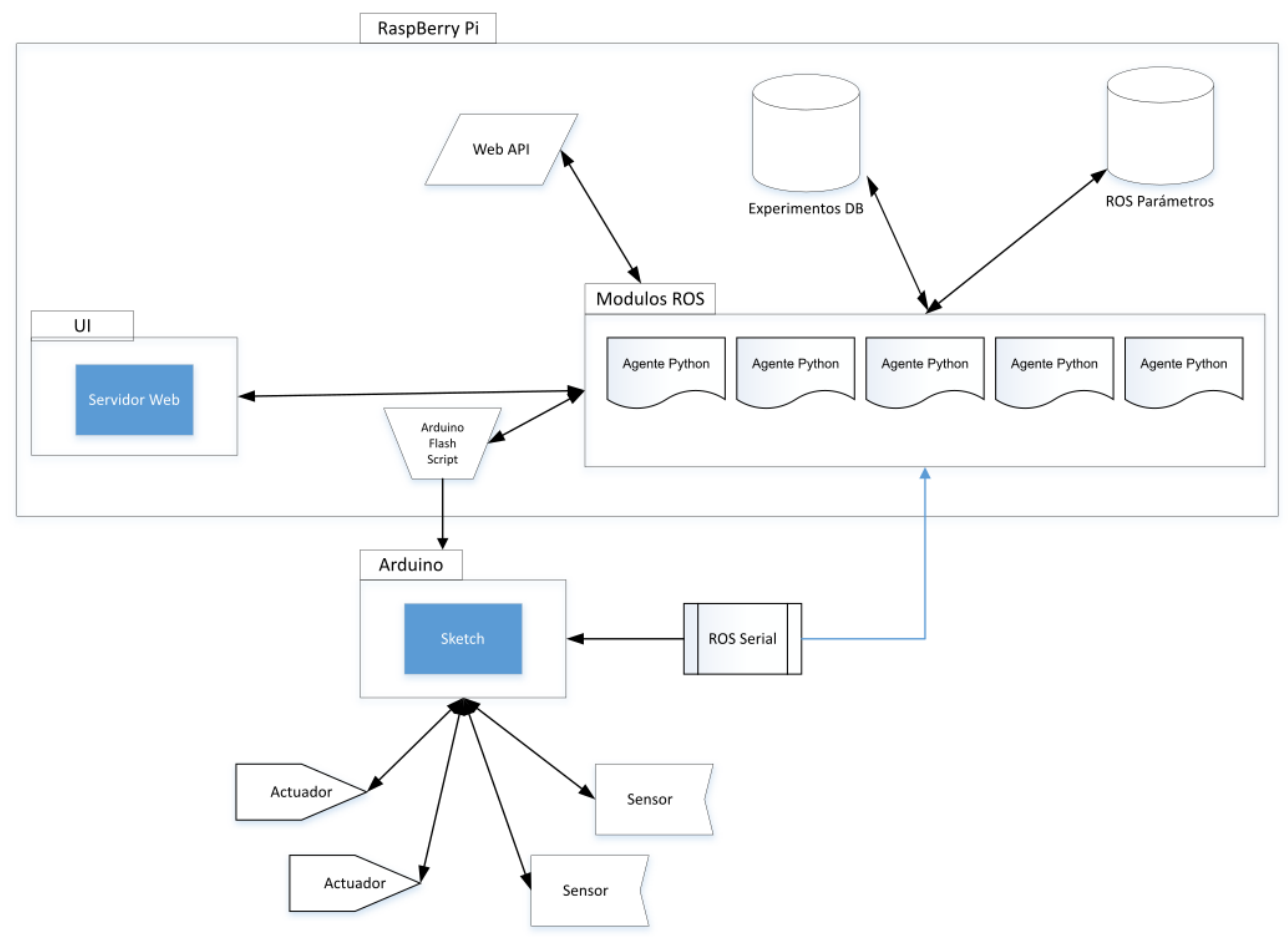

Figura 4: Arquitectura de hardware y software del SCADA. 


\section{DISCUSIÓN Y CONCLUSION}

La red de sensores inteligentes propuesta para la automatización de una planta de desalación de agua exige el estudio y aplicación de nuevas tecnologías para la medición inteligente de datos en tiempo real, la gestión de recursos y su supervisión operativa.

En este trabajo se describe la arquitectura propuesta para la gestión de una WSN de bajo coste para monitoreo continuo del comportamiento de una planta de desalinización. El sistema tiene como objetivo proporcionar mediciones continuas del proceso producción de agua. Para ello, se presenta una arquitectura de código abierto tanto para el hardware como el software. El sistema utiliza sensores gestionados por placas Arduinos con el objetivo de proporcionar mediciones continuas del proceso y comunicarlas al sistema de gestión central. Para la adquisición de datos, se emplea una plataforma basada un Sistema Multi-Agente utilizando un framework denominado SPADE que permite integrar no solo los agentes reactivos propuestos sino, además la futura incorporación de agentes para el control inteligente de la planta. Para garantizar la comunicación entre la computadora Raspberry PI y las unidades de control de sensado (Arduino) se empleará el framework denominado ROS. La elección de usar un framework como ROS se debe a que se pretende apuntar a un desarrollo futuro predecible en la automatización del proceso, y para ello ROS es una opción sostenible en su evolución.

Los resultados preliminares de las pruebas de concepto demostraron que, con la calibración adecuada, el sistema propuesto tiene la capacidad de medir continuamente los parámetros de calidad del proceso y transmitirlos a una base de datos en tiempo real, a un costo razonable y utiliza tecnologías que pueden ser asequibles a nivel de investigación.

Trabajos futuros, incorporarán el control inteligente de la planta, así como el análisis de datos del proceso que permitan identificar patrones óptimos de operación y predecir conductas anómalas en tiempo real.

\section{AGRADECIMIENTOS}

Los autores agradecen al equipo de trabajo del Laboratorio de Tecnologías Emergentes (LabTEm) y a la Universidad Nacional de la Patagonia Austral, por la colaboración y por todo el aporte realizado en el desarrollo de este trabajo.

\section{REFERENCIAS}

ABBAS, H., SHAHEEN S., AMIN M. (2015). "Simple, Flexible, and Interoperable SCADA System Based on Agent Technology"; Journal of Intelligent Control and Automation, 2015, 6, pp 184-199. Published Online August 2015 in SciRes.

AHSAN K., SHAH y KINGSTON P. (2010) "RFID Applications: An Introductory and Exploratory Study”; IJCSI International Journal of Computer Science Issues, Vol. 7, Issue 1, No. 3, January 2010.

ALMAZYAD A. S., SEDDIQ Y. M., ALOTAIBI A.M., AL-NASHERI A.Y., BENSALEH M.S., OBEID A. M. and QASIM S. M. (2014). "Proposed Scalable Design and Simulation of Wireless Sensor Network-Based Long-Distance Water Pipeline Leakage Monitoring System ”; Journal of Sensors 2014, 14(2), pp 3557-3577. 
ARAAR A., and KHALI H. (2012). "Investigating RFID Tags Fusion in a Clustered WSN Applied to Vehicle Identification and Tracking”; (2012) International Journal of Computing and Information Technology, 1, pp 17-24.

BARRET STEVEN F. (2013). “Arduino Microcontroller Processing for Everyone!'. Third Edition, Morgan \& Claypool Publishers (C2013.

BOYLE T., GIURCO D., MUKHEIBIR P., LIU A., DELANEY C., WHITE S., STEWART R. (2013). Intelligent Metering for Urban Water: A Review. Water. 5. pp 1052-1081.

CAFFERY J., y GORDON L. (2010). "Subscriber Location in CDMA Cellular Network"; (2010) IEEE Transactions on Vehicular Technology, 47, pp 406-416.

CALLAWAY E.H. (2004). "Wireless Sensor Networks: Architectures and Protocols"; (2004) CRC Press, Boca Raton.

CHARLEBOIS P. O. (2004). "Radio Frequency Indetification (RFID): Principles and Applications. Electromagnetic Fields and Waves"; (2004) McGill University, Montreal.

CORUCCI F., ANASTASI G. and MARCELLONI F. (2011). "A WSN-based testbed for energy efficiency in buildings, Computers and Communications". (ISCC), 2011 IEEE Symposium on, Kerkyra, 2011, pp. 990-993.

ENGLUND C., and WALLIN H. (2004). "RFID in Wireless Sensor Network”. Technical Report, Department of Signals and Systems, Chalmers University of Technology, Sweden, April 2004.

GUERRIERI A., RUZZELli A., FORTINO G., and O'HARE G. (2011). “A WSN-based building management framework to support energy-saving applications in buildings". (2011); Advancements in Distributed Computing and Internet Technologies: Trends and Issues, Al-Sakib Khan Pathan, Mukaddim Pathan, Hae Young Lee, eds, pp 161-174.

GURURNG T. R., STEWEART R. A., BEAL C. D., SHARMA A. K., (2015) “Smart meter enabled water end-use demand data: platform for the enhanced infrastructure planning of contemporary urban water supply networks", Journal of Cleaner Production, Volume 87, pp 642-654.

HANI A. and KOIV T. (2012). "Energy Consumption Monitoring Analysis for Residential, Educational and Public Buildings". (2012) Smart Grid and Renewable Energy, Vol. 3 No. 3, 2012, pp. 231-238.

HUANG X., YI J., CHEN S. and ZHU X. (2015). "A Wireless Sensor Network-Based Approach with Decision Support for Monitoring Lake Water Quality”. (2015) Journal of Sensors, 15(11), pp 29273-29296.

JIANG P., XIA H., HE Z. and WANG Z. (2009). "Design of a Water Environment Monitoring System Based on Wireless Sensor Networks”. (2009) - Journal of Sensors, 9(8), pp 6411-6434.

LUCK M. (1999). “From Definition to Deployment: What Next for Agent-Based Systems?”. The Knowledge Engineering Review, (1999) 14, pp 119-124.

MA X., CUI R., SUN Y., PENG C. and WU Z. (2010). "Supervisory and Energy Management System of large public buildings". Mechatronics and Automation (ICMA), 2010 International Conference on, Xi'an, 2010, pp. 928-933.

MELGAR E. and DIEZ C. (2012). Arduino and Kinect Projects: Design, Build, Blow Their Minds. Apress Berkely, CA, USA @ 2012.

NARAYAN S. P., ASSAF M. H., PRASAD S. K. (2015). “Wireless Sensor Enabled Public Transportation System”. International Journal Communications, Network and System Sciences, 2015, 8, pp 187-196 Published Online May 2015 in SciRes.

NGUYEN K. A., STEWART R. A., ZHANG H., SAHIN O., SIRIWARDENE N., (2018) Re-engineering traditional urban water management practices with smart metering 
and informatics, Environmental Modelling \& Software, Volume 101, 2018, pp 256267.

QUIGLEY M., CONLEY K., GERKEY B. P., FAUST J., FOOSTE T., LEIBS J, WHEELER R., and ANDREW Ng, (2009). ROS: an open-source Robot Operating System. ICRA Workshop on Open Source Software. Vol. 3, No. 3.2, pp 5.

SCHWIEREN J. and VOSSEN G. (2009). "A Design and Development Methodology for Mobile RFID Applications based on the ID-Services Middleware Architecture". IEEE Computer Society, (2009), Tenth International Conference on Mobile Data Management: Systems, Service and Middleware. pp. 260-266.

VENKATESAN L., SHANMUGAVEL S., and SUBRAMANIAM C. (2013). "A Survey on Modeling and Enhancing Reliability of Wireless Sensor Network". Journal of Wireless Sensor Network; (2013), 5, pp 41-45.

ZHANG Y. (2009). "RFID-Based Tracking in Supporting Real-Time Urban Traffic Information”. (2009), IEEE 5th International Joint Conference on INC, IMS and IDC, Seoul, 25-27 August 2009, pp 657-665. 